\title{
Motor unit estimations in small muscles of the hand
}

\author{
R. E. P. SICA ${ }^{1}$, A. J. MCCOMAS, A. R. M. UPTON, \\ AND D. LONGMIRE \\ From the Department of Clinical Neurosciences, McMaster University Medical Centre, \\ Hamilton, Ontario, Canada
}

SUMMARY A quantal method has been employed to determine the numbers of functioning motor units in small muscles of the hand. In healthy subjects below the age of 60 years the mean number of hypothenar motor units was $380 \pm 79$ and the mean number of thenar units innervated by the median nerve was $340 \pm 87$. In older subjects there was a progressive reduction in the numbers of functioning units in both muscle groups; in some of these subjects the population of motor units in the extensor digitorum brevis was also estimated for comparison. A study was also performed on patients diagnosed as having generalized denervating diseases or with lesions of peripheral nerves and cervical roots; in every instance the number of functioning motor units was markedly reduced.

A quantal method for estimating the number of functioning motor units in a human muscle was described by McComas et al. (1971d). Because it possessed certain favourable features, the muscle eventually selected by these workers for this type of analysis was the extensor digitorum brevis (EDB). It was observed that the electrical activity of this muscle could be recorded with little interference from that of other muscles and that, because the muscle was reasonably thin, the sizes of the recorded motor unit potentials were less likely to be affected by the positions of the units within the muscle belly. A third advantage in using this muscle was that it only had one endplate zone and hence the evoked motor unit potentials recorded by an overlying electrode could summate algebraically. The assumptions inherent in the quantal method were described by McComas et al. (1971d) and attempts were made to justify them. After results had been obtained by this method in healthy subjects the opportunity arose to employ it for the study of patients with various neurological disorders including chronic denervation (McComas et al., 1971e), myasthenia gravis (McComas et al., $1971 \mathrm{~b})$ and the different types of muscular dystrophy (McComas et al., 1971c; McComas et al., 1971a; Sica and McComas, 1971). The

\footnotetext{
1 On leave from the Hospital Ramos Mejia, Buenos Aires.
}

quantal technique was also used to analyse the nature of the process responsible for the wasting of muscles which occurs in old age (Campbell and McComas, 1970; Campbell et al., 1973). In spite of the large number of control subjects in whom the EDB muscle had been investigated, the choice of this muscle for studies in disease might have been criticized in view of subsequent findings by Jennekens et al. (1972). These authors described pathological changes suggestive of denervation in post mortem specimens of muscle from young subjects killed in accidents and suggested that such factors as the wearing of tight shoes could have been responsible for injury to the deep peroneal nerve. Similarly damage to this nerve (from crossing the legs) was considered by Hayward (1971) to be the explanation for electromyographic features of denervation in the tibialis anterior muscle. We had already pointed out that, although it was possible that denervation might occur in the EDB muscles of some healthy subjects, it was unlikely to cause a significant fall in the motor unit population below the age of 60 years (McComas et al., 1971d). Nevertheless, the findings of Jennekens et al. (1972) stimulated us to re-examine the feasibility of using additional muscles for the estimation of motor unit numbers. Our search has included the brachioradialis, latissimus dorsi, sartorius, tibialis 
anterior, and soleus muscles, as well as the thenar and hypothenar muscle groups. Of these muscles the last two groups have proved suitable for the quantal method and the results obtained in healthy subjects are reported here. As part of the present investigation, motor unit populations have been determined in the muscles of elderly subjects and the values have been compared with those for EDB in the same individuals. In

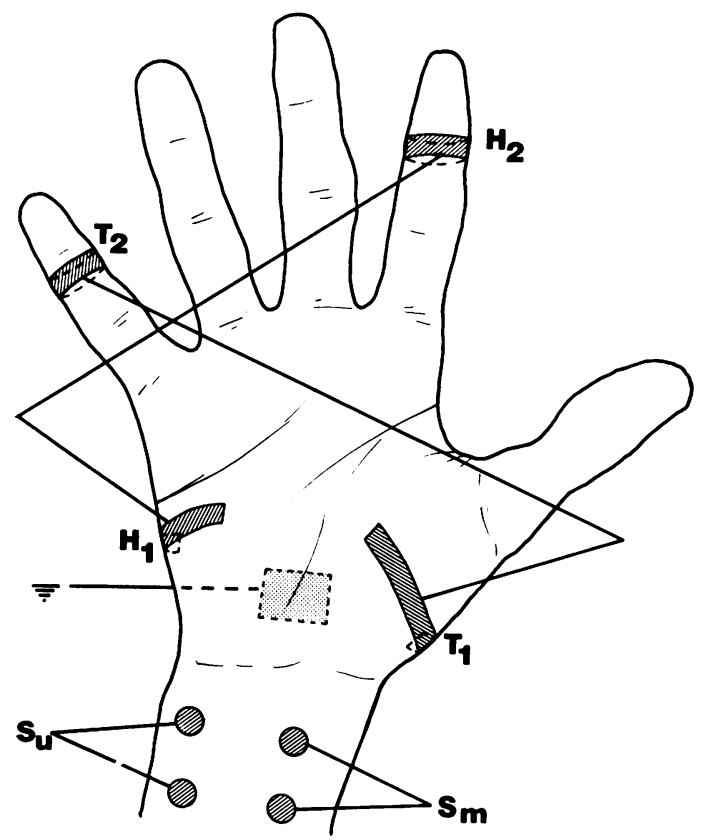

FIG. 1. Arrangement of electrodes for estimating numbers of thenar or hypothenar units. $\mathrm{S}_{\mathrm{u}}$, ulnar nerve stimulating electrodes (cathode distal); $\mathrm{S}_{\mathrm{m}}$, median nerve stimulating electrodes (cathode distal); $\mathrm{H}_{1}$ and $\mathrm{H}_{2}$ are stigmatic and reference recording electrodes for hypothenar studies; $\mathrm{T}_{1}$ and $\mathrm{T}_{2}$ are corresponding electrodes for thenar muscles. See text.

addition, the sensitivity of the thenar and hypothenar motor unit estimations has been tested by examining patients with traumatic lesions of the median or ulnar nerves, or of the cervical roots. Other patients with widespread neuropathic disorders have also been studied. A similar investigation of the thenar muscles has recently been reported by Brown (1972) and the results of the two studies have been compared.
METHODS

SUBJECTS A total of 92 subjects were examined, of whom 43 were male and 49 female; their ages ranged from 5 to 97 years. All the subjects were judged to be in good health for their age and even the eldest ones led active lives. None had any evidence of neurological disease or of a metabolic disorder-for example, diabetes, renal failure.

STIMULATING AND RECORDING CONDITIONS Surface electrodes were used for nerve stimulation and for recording the muscle responses. The stimulating electrodes were chlorided silver discs, $10 \mathrm{~mm}$ in diameter, which were mounted in a Perspex holder so that their centres were $3 \mathrm{~cm}$ apart. The stigmatic and reference recording electrodes were strips of silver foil, $6 \mathrm{~cm} \times 6 \mathrm{~mm}$, which were fastened to the skin by adhesive tape. The earth electrode was a lead plate which was fixed to the back of the hand. For studying hypothenar motor units the stimulating electrodes were placed over the ulnar nerve at the wrist. The stigmatic electrode was bent so as to lie transversely across the hypothenar eminence and round the ulnar border of the hand at the level of its maximum convexity (Fig. 1). The reference electrode was wrapped around the distal part of the index finger; the choice of this finger rather than the ring or little fingers eliminated the possibility of mistaking antidromically-conducted sensory nerve potentials in digital branches of the ulnar nerve for a motor unit potential.

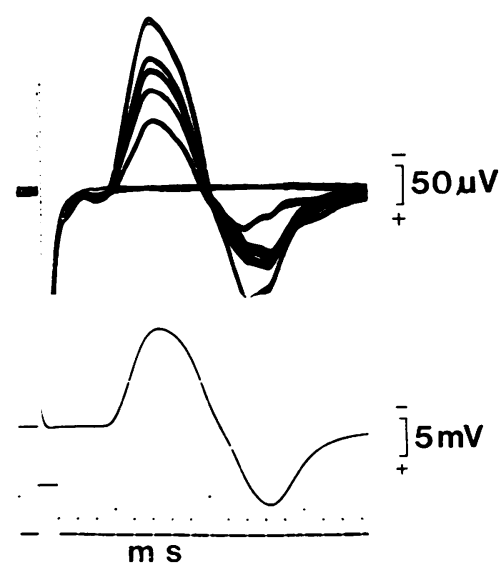

FIG. 2. Estimation of number of median-innervated thenar motor units in a 16 year old control subject. Top shows first five incremental responses as stimulus increased from subthreshold intensity. Bottom shows maximal $M$ wave of muscle. Details of calculation given in text. 
For investigating the thenar muscles, the stimulating electrodes were placed over the median nerve at the wrist. The curved stigmatic recording electrode was placed round the proximal part of the thenar eminence as shown in Fig. 1. To avoid inadvertent recording of the antidromically-conducted sensory nerve potential the reference electrode was attached to the distal part of the little finger.

The stimuli consisted of rectangular voltage pulses, $50 \mu \mathrm{sec}$ in duration, delivered from a Devices Ltd Model 3072 stimulator which itself received a triggering pulse from a digital timing device (Devices Ltd Digitimer, Model 3290). The responses were fed through a specially designed low-noise amplifier, using a frequency response which was $3 \mathrm{db}$ down at $2 \mathrm{~Hz}$ and $10 \mathrm{kHz}$. The responses were displayed and measured on a storage oscilloscope with variable persistence (Hewlett-Packard Ltd., Model 141 B).

\section{RESULTS}

QUANTAL RESPONSES Figure 2 (upper) shows the way in which the thenar muscle responses
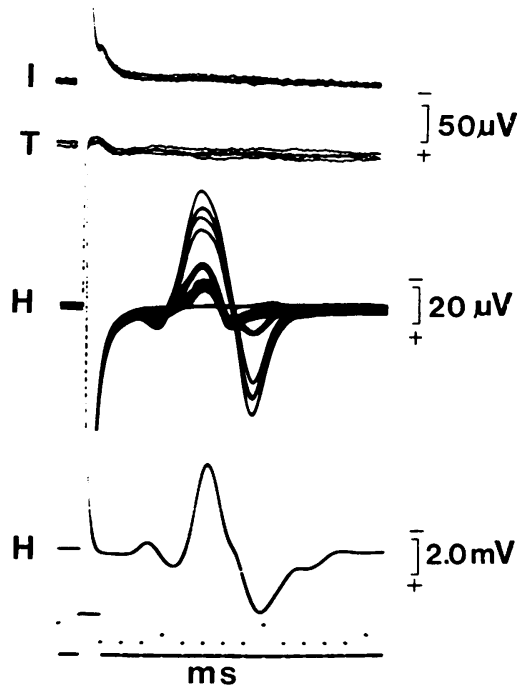

FIG. 3. Estimation of number of hypothenar motor units in a 39 year old control subject. The top three traces show that, after weak stimulation of the ulnar nerve, incremental responses can be recorded from the hypothenar $(\mathrm{H})$ group, while no activity could be detected over the thenar $(\mathrm{T})$ or first dorsal interosseous (I) muscles. In reproduction the two hypothenar incremental responses with lowest thresholds appear as one; altogether seven increments are present. Mean increment size, $21 \mu \mathrm{V}$; maximum $M$ wave, $8.6 \mathrm{mV}$; estimated number of units, 410 . grew larger in discrete increments as the stimuli were increased from a subthreshold intensity. Each of these increments was attributed to the excitation of an additional motor unit and it was usually possible to discriminate between eight and 12 such steps with reasonable certainty. With a stronger but constant stimulus the amplitudes of the responses fluctuated considerably. This phenomenon probably reflected the ways in which motor axons with similar thresholds could fire in different combinations ('alternation'; see McComas et al., 1971d). In Fig. 2 it can be seen that the first five response increments corresponded to a total amplitude of $360 \mu \mathrm{V}$, measured from peak to peak; hence the mean amplitude of these five units was $72 \mu \mathrm{V}$. When a supramaximal stimulus was given to the median nerve a maximal response (' $M$ wave') with an amplitude of $22.5 \mathrm{mV}$ was evoked (Fig. 2 , lower). Hence the estimated number of motor units was 313. Similarly, for the hypothenar muscles, the number of units estimated from Fig. 3 was 410 .

Both these estimates are, of course, gross approximations even when, as in an actual experiment, the sizes of the motor units samples are doubled - that is, $8-12$ units. The assumptions inherent in the quantal method for estimating motor unit populations have already been considered in detail in relation to the EDB muscle (McComas et al., 1971d). They are:

FIRST ASSUMPTION That the electrical activity is derived from a single muscle This is clearly not the case in the present study for, of the thenar muscle group, the median nerve innervates the abductor pollicis brevis and opponens pollicis as well as part of the flexor pollicis brevis. This nerve also supplies the first two lumbrical muscles. We have assumed that the contribution of the relatively small lumbrical muscles to the evoked activity is slight; the greater distance of these muscles from the recording electrodes, compared with the thenar muscle group, should also minimize their contributions. In order to obtain as good an average as possible of the electrical activity in the thenar muscles, we have employed a large surface electrode which extended over half of the circumference of the combined muscle bulk. When a maximal stimulus was used at the wrist it was necessary to make 
sure that the current had not spread sufficiently to excite motor axons in the ulnar nerve, thereby evoking responses in the ulnar-innervated thenar muscles (adductor pollicis and part of the flexor pollicis brevis). In most adult subjects the relationship between stimulus strength and the amplitude of the $M$ wave recorded over the thenar muscles showed a definite plateau such that a $30-50 \%$ increase in stimulus strength caused no augmentation of the response. Stimuli larger than this did produce a further increase in the response and it could be shown that this was due to excitation of the ulnar nerve. In children or adults with slender wrists the plateau in the stimulus-response curve was often illdefined. In such cases we have preferred to base the calculation of motor unit number on the maximum thenar response after stimulation of the median nerve in the antebrachial fossa rather than at the wrist. At the former site the possibility of stimulus spread to the ulnar nerve was negligible because of its remoteness. In such subjects the extra length of median nerve involved would have resulted in a rather smaller $M$ wave (typically $10 \%$ ) than that evoked by nerve stimulation at the wrist, due to increased temporal dispersion of the motor unit potentials. No correction has been made for this factor in the results. Sometimes, however, the thenar response was larger when the median nerve was stimulated at the elbow instead of the wrist. This finding was not investigated further and has been attributed to the presence of a communicating branch running from the median to the ulnar nerve in the forearm, and thence to the thenar muscles. When this situation existed the amplitude of the response after stimulation at the wrist was employed in the calculation of motor unit population.

In the study of the hypothenar motor units, four muscles are involved-the abductor, flexor and opponens digiti minimi, and the palmaris brevis, and each receives its innervation from the ulnar nerve. This nerve also supplies all the interossei, the medial two lumbricals, the adductor pollicis, and part of the flexor pollicis brevis. Fortunately, of all the motor branches of the ulnar nerve, that to the hypothenar muscles has the lowest threshold, provided care is taken in placing the stimulating electrodes correctly at the wrist. This point is demonstrated in Fig. 3 in which it can be seen that there is no response from the first dorsal interosseous and thenar muscles even though several units have been activated within the hypothenar group of muscles. To avoid contamination of the maximum $\mathbf{M}$ wave with activity evoked in median-innervated muscles of the hand, the amplitude of the response was checked against that resulting from stimulation of the ulnar nerve at the elbow.

SECOND ASSUMPTION The incremental responses evoked by graded stimulation correspond to the activation of single units This assumption was discussed in some detail by McComas et al. (1971d) in relation to EDB and the same considerations apply to the thenar and hypothenar muscles. A major problem in using the latter muscle groups for quantal studies is that the thresholds of the most excitable motor axons at the wrist are closer together than are those of the EDB motor axons in the peroneal nerve at the ankle. Consequently, the chances of 'alternation' occurring (see above) are considerable and, of course, increase as the stimulation intensity is raised. In our experience, it has not been possible to obtain a satisfactory sample of motor unit potentials in about $5 \%$ of the normal population because of this difficulty.

THIRD ASSUMPTION Evoked motor unit potentials summate algebraically Although several muscles are involved in both the thenar and hypothenar estimations of motor unit populations each muscle, because of its small size, probably has only one end-plate zone. In the case of the opponens pollicis, this is known to be so (Christensen, 1959). In each of two preliminary experiments an attempt was made to locate the greatest density of end-plate zones by mapping the field potentials developed during muscle excitation. In these experiments supramaximal stimuli were delivered to the appropriate nerve, median or ulnar, and the muscle responses were recorded with a chlorided silver ball electrode in contact with the skin overlying the muscle belly. In Fig. 4 it can be seen that over the thenar muscles there was one clearly defined region (electrode positions $4,5,6$ ) in which the evoked muscle action potential displayed a large initial negativity, indicating the presence of an end-plate rich zone underneath. With the stig- 


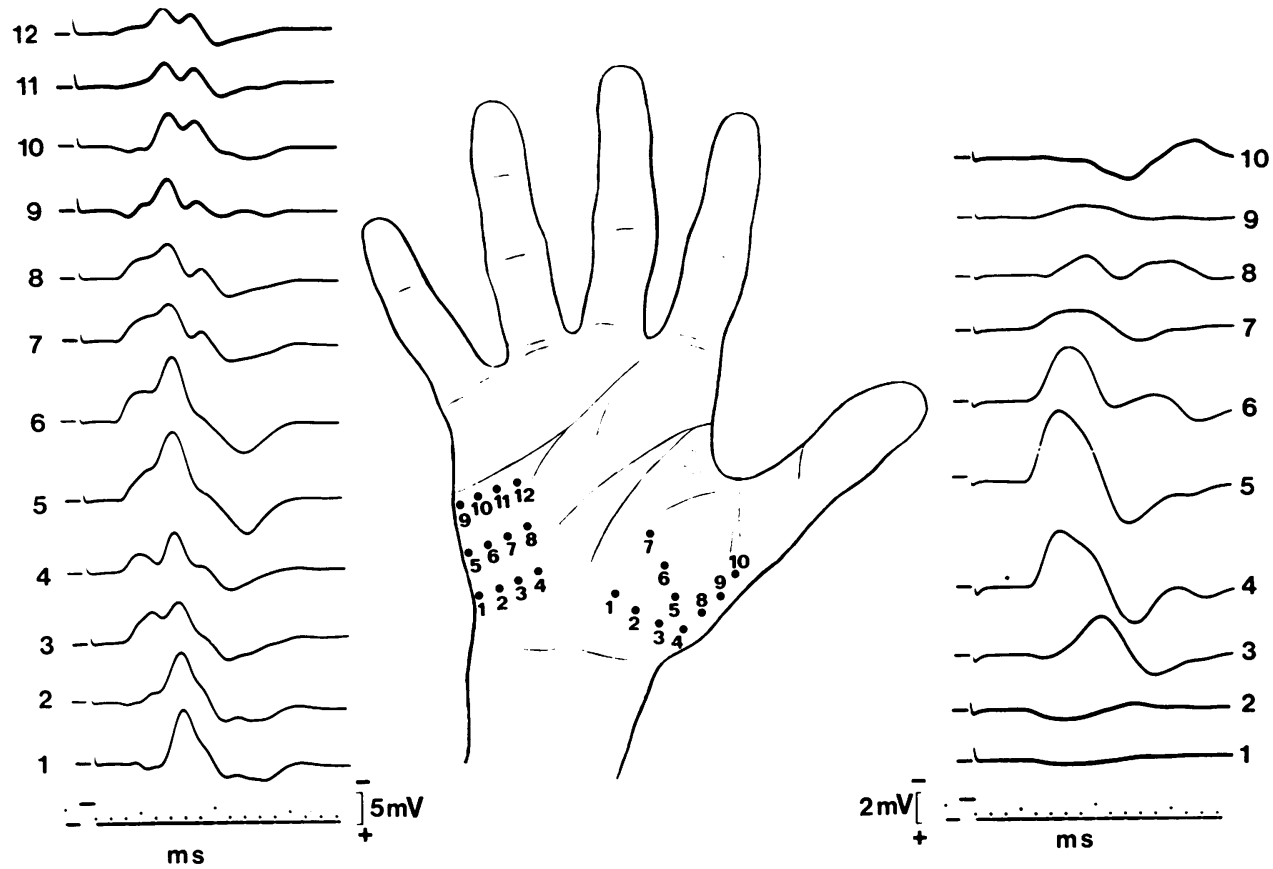

FIG. 4. Responses recorded with a small exploring electrode at different points over the thenar and hypothenar eminences, after stimulation of the median or ulnar nerve respectively. The reference electrodes were in the same positions as in Fig. 1. See text.

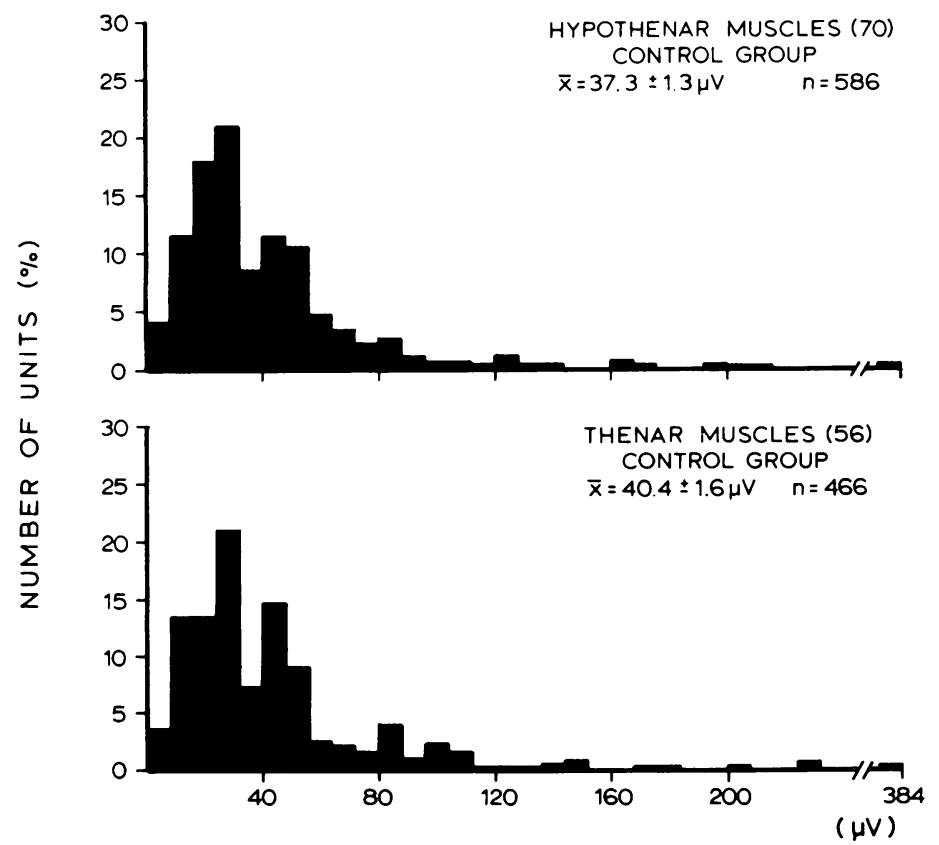

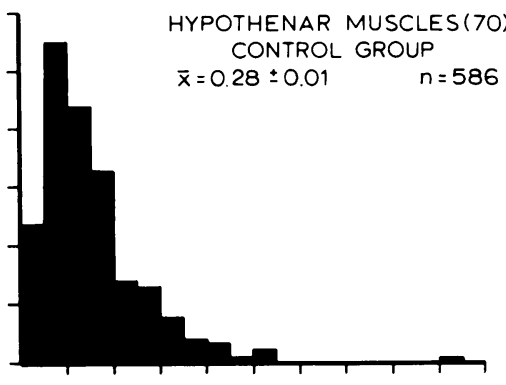

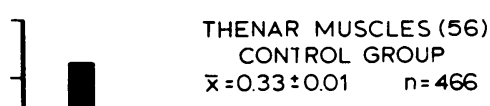

MOTOR UNIT POTENTIAL AMPLITUDE

FIG. 5. Amplitudes of motor unit potentials (response increments) in thenar and hypothenar muscles of control subjects aged 15 to 60 years. Results have been expressed in both absolute and relative values (left and right histograms respectively). Means given with standard errors. 
matic silver strip electrode positioned as described in the Methods section (see also Fig. 1), the successively recruited motor unit potentials nearly always summated algebraically. In contrast, the mapping experiment disclosed a more widely distributed initial negativity over the hypothenar muscles. Although a large strip electrode was employed in the motor unit estimating studies, the evoked hypothenar responses frequently had complex forms and sometimes displayed an initial positivity. On the basis of the mapping experiments, it seemed probable that the end-plate zones of the hypothenar muscles were widely separated but it was also possible that appreciable differences in nerve fibre length might have contributed to the irregular form of the potentials.

FOURTH ASSUMPTION The motor unit potentials used in the calculation of the mean potential amplitude are representative of those generated by the total population of units Figure 5 shows the sizes of all the motor unit potentials sampled in the 39 subjects who were aged between 15 and 60 years. It can be seen that in the thenar population the values ranged from 2 to $380 \mu \mathrm{V}$, or from 0.01 to $1.81 \%$ of the maximum $M$ waves in the subjects concerned. For the hypothenar units the sizes of the potentials ranged from 2 to $365 \mu \mathrm{V}$,

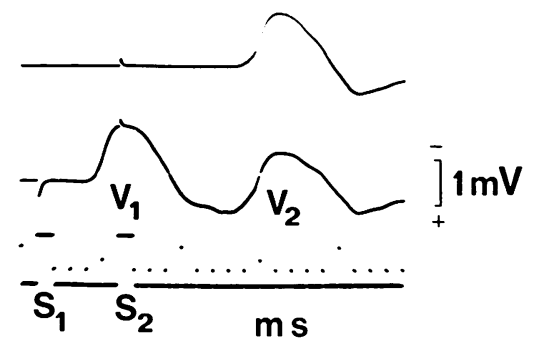

FIG. 6. Results of collision experiment using submaximal stimulation of median nerve at wrist $\left(\mathbf{S}_{1}\right)$ and at elbow $\left(\mathrm{S}_{2}\right)$; corresponding responses of thenar muscles are $\mathrm{V}_{1}$ and $\mathrm{V}_{2}$. In the top trace only $\mathrm{S}_{2}$ was delivered. In the bottom trace $\mathrm{S}_{1}$ preceded $\mathrm{S}_{2}$ by 5 msec; the small (ca. $22 \%$ ) reduction in $\mathrm{V}_{2}$ amplitude indicated that impulse collision was occurring in only a small population of the excited axons. Therefore most of the axons with lowest thresholds at the wrist form a different population to the corresponding units at the elbow (see text). or from 0.01 to $1.83 \%$ of the corresponding $\mathrm{M}$ waves (Fig. 5). The use of the maximum $M$ wave as a reference for potential sizes, rather than absolute values of amplitude, had the advantage of removing the effects of varying skin thicknesses and inadvertent differences in electrode placement among the population of subjects studied.

The possibility of a systematic error in the sampling of motor units was tested by comparing the response amplitudes of the most, and the least, excitable units within each sample of 8-12 units. In the 56 thenar muscles investigated the mean potential amplitudes of the most and least excitable motor units were the same $(0.35 \%$ of the $M$ wave) and hence it did not appear that the stimuli were preferentially exciting axons belonging to the larger motor units. In the 70 hypothenar muscles, the lowest-threshold units tended to have smaller potential amplitudes $(0.26 \pm 0.18 \%$ of the $M$ wave, compared with $0.33 \pm 0.18 \%$ for the least excitable units; $\mathbf{P}=<0.05)$. The reasons for this difference were not apparent. However, one factor which was clearly important in determining excitability was the position of the axons relative to the stimulating electrodes. Suppose that this had not been so and that the major factor contributing to excitability had been some biophysical property of the axons themselves. In these circumstances the same axons should have the lowest thresholds irrespective of the site of stimulation. That this was not the case was shown by a collision experiment in which the median nerve was stimulated at the wrist and elbow (Fig. 6; see also McComas et al., 1971d). Each stimulus was made submaximal and excited approximately $20 \%$ of the motor axons. If the two stimuli had excited the same population of axons then impulse collision should have occurred when the shocks were delivered simultaneously or within a few milliseconds of each other. In fact, Fig. 6 shows that little collision occurred and a similar result could be demonstrated for the ulnar nerve.

REPRODUCIBILITY OF RESULTS In one subject estimations of the numbers of motor units were made on 10 different days; the values ranged from 233 to 320 with a mean of $269 \pm 29$ (SD) units. For hypothenar units investigated in the 


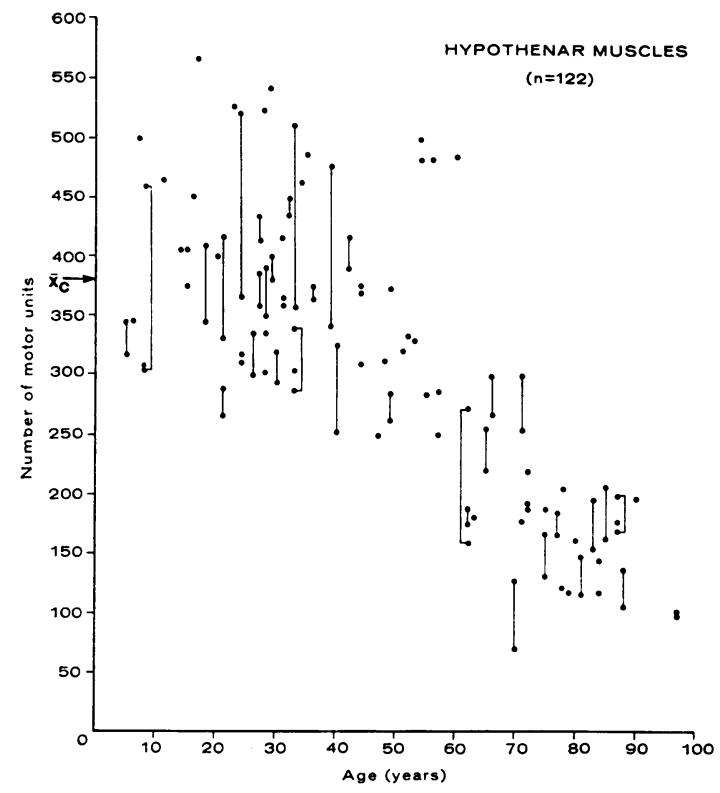

FIG. 7a. Numbers of hypothenar motor units in control subjects of different ages. Paired observations linked by vertical lines $\bar{X}_{\mathrm{c}}$, mean value for subjects under 60 (380 \pm 79 units).

same subject the largest and smallest values were 520 and 400 and the mean was $453 \pm 43$ units.

MOTOR UNIT POPULATIONS AT DIFFERENT AGES Figure 7 shows the numbers of hypothenar motor units estimated in 86 subjects aged from 5 to 97 years; a total of 122 estimations were made, since in some subjects both hands were investigated. On first inspection, it appears that there was a progressive loss of units throughout life. This is an illusion, however, and can be recognized as such if the values for elderly subjects at the right of the Figure are ignored. Indeed, statistical analysis of the results revealed that there was no significant loss of units below the age of 60 years, the correlation coefficient with age being only $0 \cdot 17$. Beyond this time, the number of units declined until rather less than half $(42 \%)$ were left by the ninth decade. The importance of the seventh decade as a critical period for the onset of ageing changes in muscle is clearly seen in Fig. 8, where the observations for each decade have been averaged.

The results for thenar muscles in 72 subjects

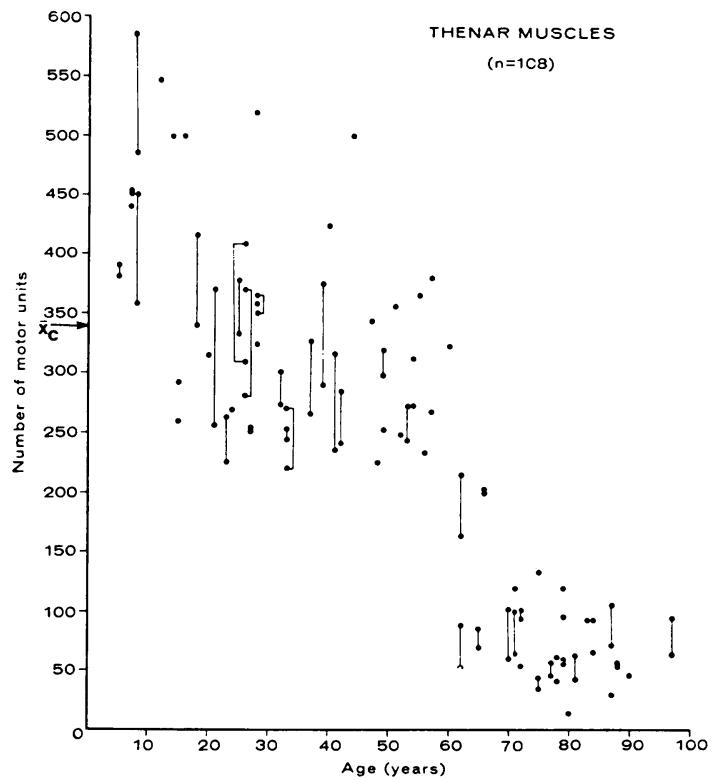

FIG. 7b. Corresponding values for median-innervated thenar motor units $\left(\bar{X}_{\mathrm{c}}=340 \pm 87\right.$ units $)$.

TABLE

MOTOR UNITS REMAINING IN EXTENSOR DIGITORUM BREVIS (EDB), THENAR AND HYPOTHENAR MUSCLES OF 12 ELDERLY SUBJECTS (14 SIDES)*

\begin{tabular}{|c|c|c|c|c|c|}
\hline \multirow[t]{2}{*}{ Subject } & \multirow[t]{2}{*}{ Sex } & \multirow{2}{*}{$\begin{array}{l}\text { Age } \\
(y r)\end{array}$} & \multicolumn{3}{|c|}{ Percent units remaining } \\
\hline & & & $E D B$ & Thenar & Hypothenar \\
\hline J.S. & F & 78 & 39 & 18 & 54 \\
\hline A.S. & $\mathbf{M}$ & 84 & 24 & 19 & 31 \\
\hline B.D. & $F$ & 87 & 23 & 9 & 47 \\
\hline R.A. & $\mathbf{F}$ & 84 & 5 & 27 & 38 \\
\hline M.M. & $\mathbf{F}$ & 72 & 32 & 16 & 58 \\
\hline \multirow[t]{2}{*}{ J.P. } & $\mathbf{M}$ & 87 & 31 & 21 & 45 (L) \\
\hline & & & 31 & 31 & $53(\mathrm{R})$ \\
\hline R.T. & $\mathbf{M}$ & 79 & 4 & 28 & 31 \\
\hline G.D. & $\mathbf{F}$ & 78 & 15 & 12 & 32 \\
\hline \multirow{2}{*}{ W.A. } & $\mathbf{M}$ & 70 & 6 & 18 & 19 (L) \\
\hline & & & 5 & 30 & 34 (R) \\
\hline M.S. & $\mathbf{F}$ & 90 & 22 & 14 & 52 \\
\hline G.P. & $\mathbf{F}$ & 71 & 31 & 35 & 47 \\
\hline H.P. & $\mathbf{M}$ & 75 & 50 & 39 & 49 \\
\hline \multicolumn{3}{|c|}{$\begin{array}{l}\text { Units remaining; mean } \% \pm S D \\
\text { Units 'lost'; } \quad \text { mean } \% \pm S D\end{array}$} & $\begin{array}{l}23 \pm 14 \\
77 \pm 14\end{array}$ & $\begin{array}{l}23 \pm 9 \\
77 \pm 9\end{array}$ & $\begin{array}{l}42 \pm 11 \\
58 \pm 11\end{array}$ \\
\hline
\end{tabular}

* The results are expressed as percentages of mean values for control subjects below the age of 60 years. 


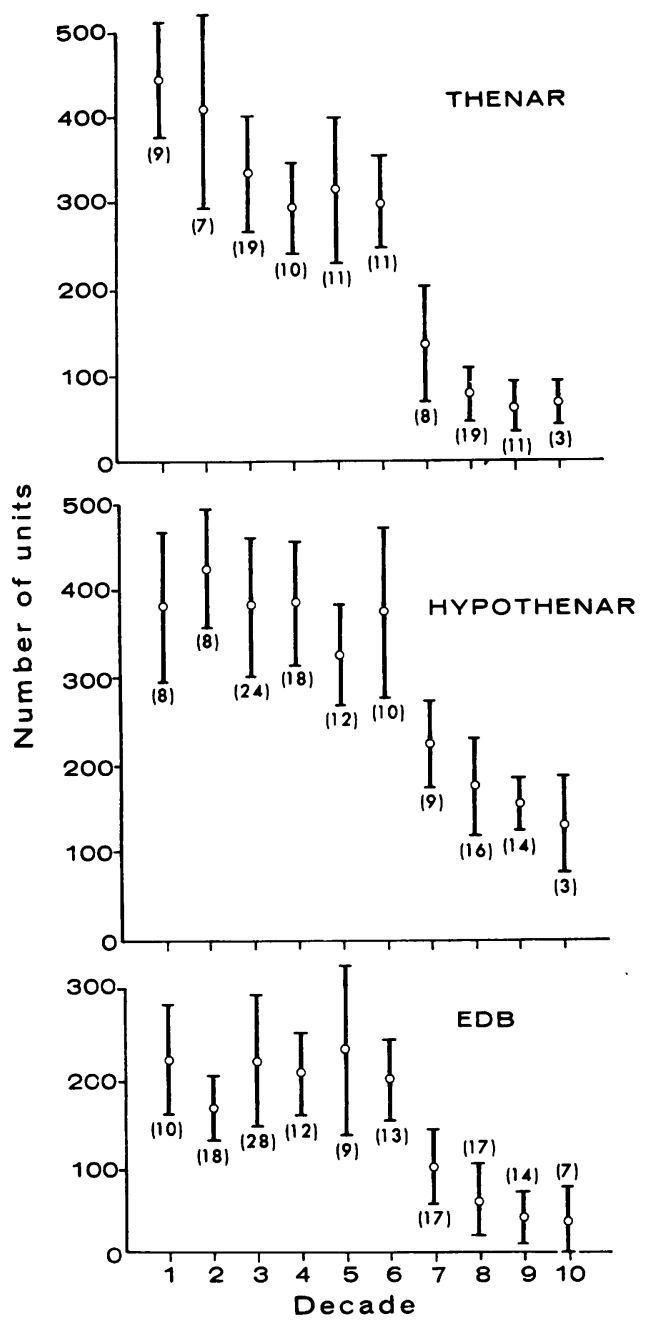

FIG. 8. Mean number of motor units for control subjects in each decade. Vertical bars represent $\pm 1 S D$; number of observations given in parentheses below. The values for the extensor digitorum brevis muscle (EDB) have also been given for comparison.

aged 5 to 97 years are also displayed in Fig. 7; altogether 108 estimations were performed. In these muscles it appeared that the motor unit population remained constant in size between 15 and $60(r=0 \cdot 20 ; P=>0 \cdot 1)$. Before the age of 15 years the numbers of units were significantly higher $(\mathrm{P}=<0.001)$; whether this was a genuine feature of the thenar innervation or a reflection of the small sample of observations was not clear. Beyond the age of 60 years a very marked loss of functioning motor units took place so that, on average, only $19 \%$ remained in the ninth decade. When the values for subjects below the age of 60 were analysed separately it was found that the mean number of thenar motor units was $340 \mathrm{SD} \pm 87(n=67)$ and that for the hypothenar units was $380 \pm 79(n=77)$.

In each of 12 elderly subjects the opportunity was taken to estimate the numbers of units in the hypothenar, thenar and EDB muscles on the same side (14 sides altogether). In Fig. 9 and the Table it can be seen that the loss of hypothenar units with age was considerably less severe than that occurring in EDB and the thenar muscles. When the results for each of the three types of estimation were pooled and expressed in terms of the corresponding mean values for younger subjects (see above), the mean percentage losses within this elderly population were 58 (hypothenar), 77 (thenar), and 77 (EDB). Thus, there was no difference between the percentage losses for the thenar and EDB muscles but the hypothenar loss was significantly less severe than either $(P=<0.001$ in each case).

SIZES OF REMAINING UNITS IN OLD AGE The amplitudes of the motor unit potentials were of considerable interest in that they reflected the sizes of the motor units, assuming that changes in muscle fibre diameter, and other factors affecting the action currents of individual fibres, were relatively small. In Fig. 10 the values obtained in the elderly subjects have been pooled and compared with those of subjects aged 15-60 years. It can be seen that there was a significant increase in the mean potential amplitudes of the surviving motor units in both thenar and the hypothenar muscles of elderly subjects $(P=<0.001$ in each case). In surveying the thenar and hypothenar results the increased potential amplitudes of the latter were especially striking in view of the smaller reduction of functioning motor units which occurred in hypothenar muscles during ageing. Thus the mean potential amplitude of the remaining hypothenar units in the subjects over 60 years was $58 \pm 44 \mu \mathrm{V}$ (SD) compared with a mean value in the younger subjects of $37 \pm 31 \mu \mathrm{V}$; the corresponding mean numbers of functioning motor units were $177 \pm 54$ and $380 \pm 79$ respectively. In the older subjects the 


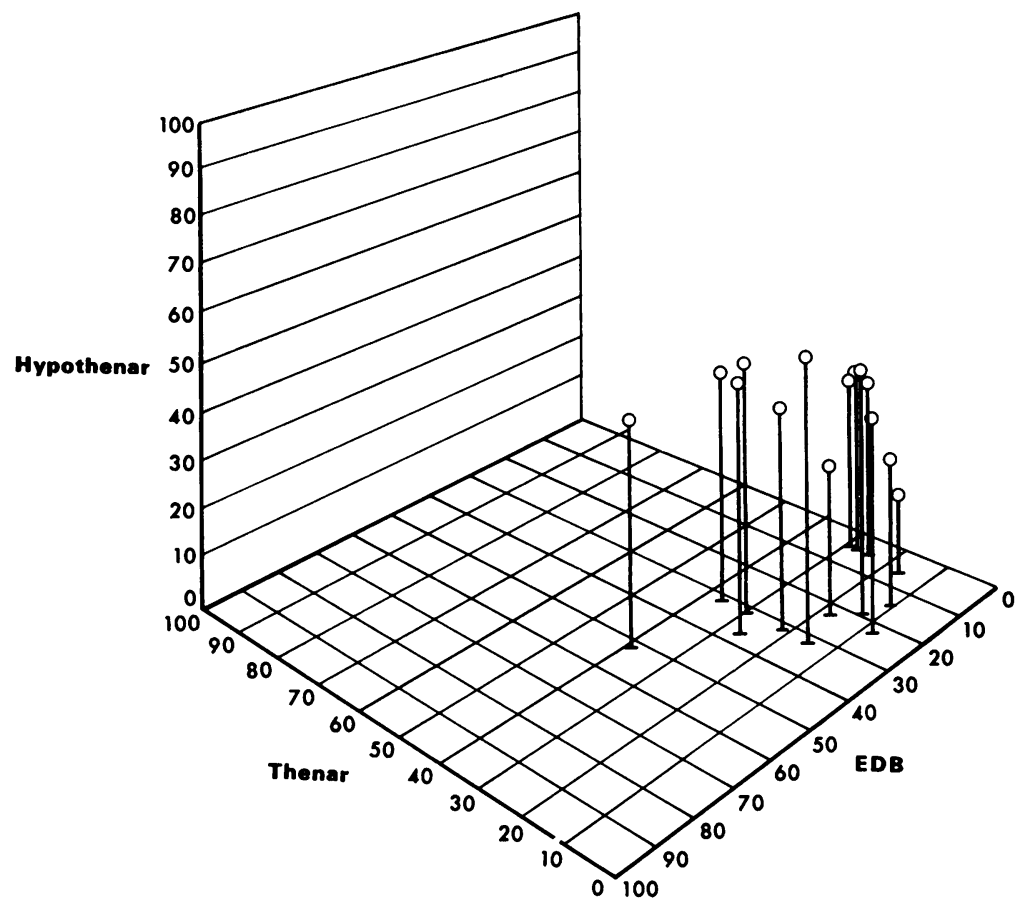

FIG. 9. Comparison of motor unit populations in thenar, hypothenar, and EDB muscles of 12 elderly subjects (14 sides). Note relative sparing of hypothenar muscles by ageing process.

PERCENT CONTROL MEAN
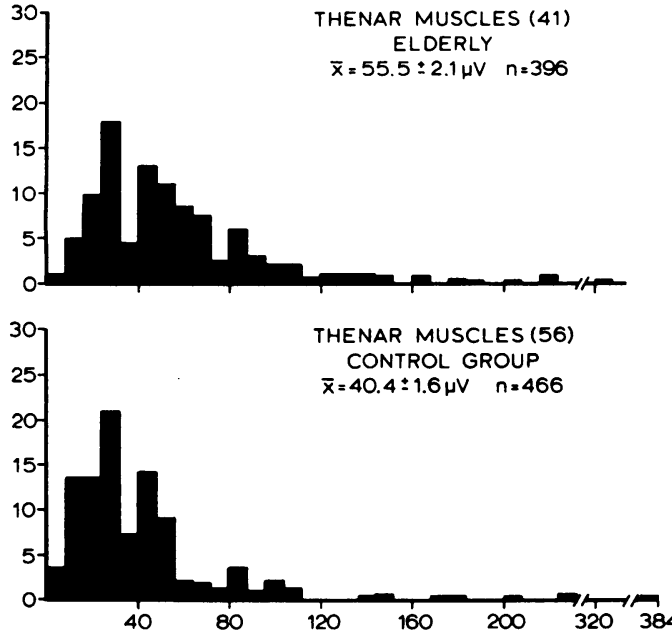

MOTOR UNIT POTENTIAL AMPLITUDE ( $\mu \mathrm{V})$
HYPOTHENAR MUSCLES (42) ELDERLY

$\bar{x}=58.4: 2.3 \mu V \quad n=388$
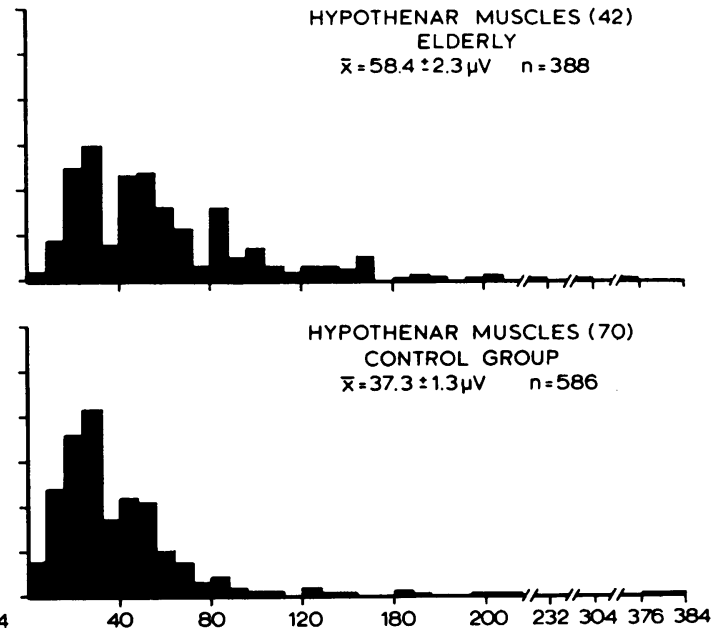

FIG. 10. Amplitudes of thenar and hypothenar motor unit potentials (response increments) in control group (below 60 years) and in elderly subjects. In this Figure the means have been given with their standard errors. 


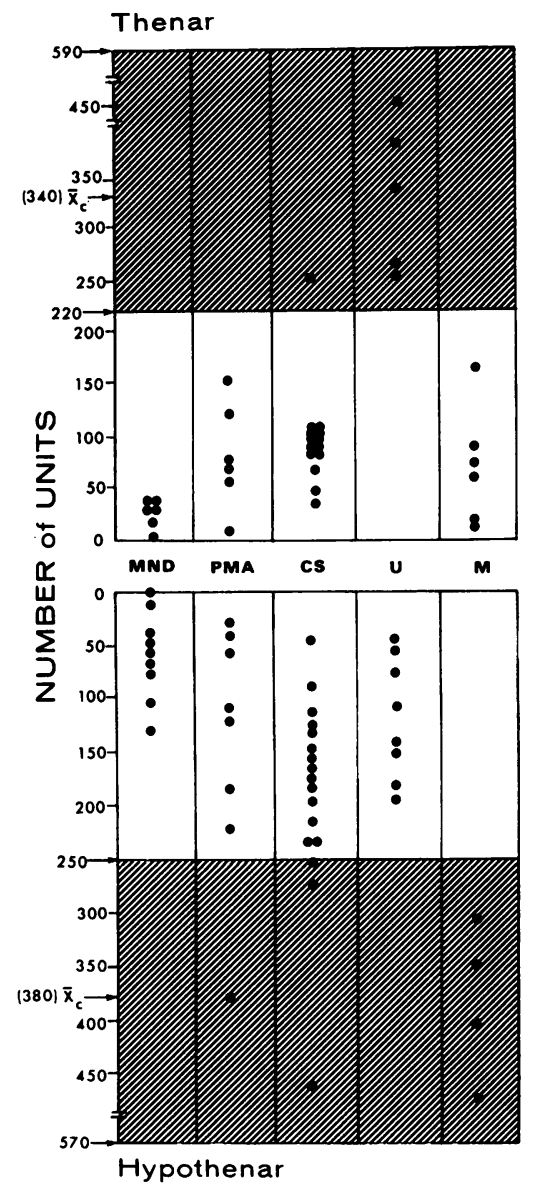

FIG. 11. Numbers of thenar and hypothenar motor units in patients under 60 years of age with motoneurone disease (MND), peroneal muscular atrophy (PMA), cervical spondylosis (CS), ulnar nerve lesions at the elbow (U), and median nerve lesions at the wrist (M). The control ranges are indicated by shaded areas; $\bar{X}_{\mathrm{c}}$, mean value. mean number of thenar units was $83 \pm 46$ and the mean potential amplitude was $56 \pm 42 \mu \mathrm{V}$; in the young subjects the respective values were $340 \pm 87$ units and $40 \pm 35 \mu \mathrm{V}$.

THENAR AND HYPOTHENAR MOTOR UNIT POPULATIONS IN DENERVATING DISORDERS Estimations of numbers of motor units in small muscles of the hand have been performed on a large number of patients with clinical and other electrophysiological evidence of denervation. In order to avoid the possibility of ageing contributing to muscle denervation, the results shown in Fig. 11 have been obtained in patients below the age of 60 years. In all these patients the electrophysiological investigations were carried out within six months of the onset of neurological symptoms. It can be seen that by this time the patients with motoneurone disease exhibited very severe reduction in the numbers of functioning motor units. More modest losses were usually observed in patients with peroneal muscular atrophy. The results in patients with carpal tunnel syndromes or lesions of the ulnar nerve at the elbow were of particular interest, for they demonstrated how one population of motor units could be affected while the other was left intact.

Finally Fig. 10 shows that, in patients with cervical spondylosis, both thenar and hypothenar motor unit populations were diminished, as would be anticipated in view of their common segmental innervation (C8 and T1). Two examples of the responses evoked in partially denervated thenar muscles are shown in Fig. 12. One patient developed a polyneuropathy after commencing disulfiram therapy for the treat-
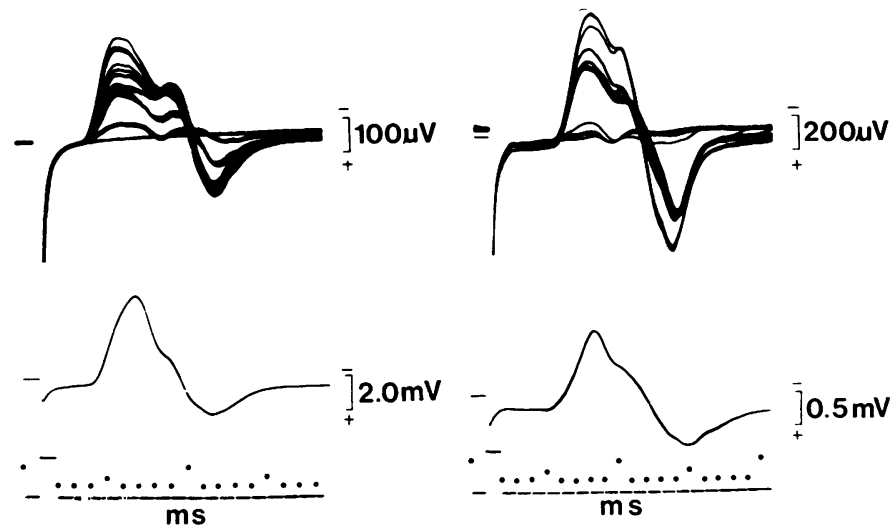

FIG. 12. Left. Thenar responses from patient with polyneuropathy (see text). At top, 9 response increments correspond to $450 \mu \mathrm{V}$, giving mean of $50 \mu \mathrm{V}$. Amplitude of maximum $M$ wave (bottom) $=8.0 \mathrm{mV}$, hence estimated number of units $=160$. Right. Thenar responses from patient with cervical root lesion. After the first 8 increments (top), the response is almost maximal (see $M$ wave at bottom). The total number of units was probably 10. 
ment of chronic alcoholism and the thenar muscles were estimated to contain 160 units (Fig. 12, left). Although this value was well below the lower limits of the normal range (220 units), the normal-sized $M$ wave $(8.0 \mathrm{mV})$ furnished electrophysiological evidence of the absence of obvious muscle wasting. In contrast, the responses shown in Fig. 12 (right) were recorded from a patient who had marked atrophy and weakness of small muscles of his hand after a neck injury. It can be seen that the motor unit population was severely depleted (10 units) and that some of the potentials generated by the surviving units were greatly enlarged, indicating the occurrence of collateral reinnervation (see Discussion). It is not the purpose of this paper to dwell upon usefulness of the motor unit estimating technique in clinical practice, and for this reason only the following instance will be described.

The patient H.F. was a 17 year old girl who, for many years, had shown some unsteadiness in walking. On clinical examination, there was no evidence of muscle wasting in the limbs nor was any sensory loss detectable, apart from impaired vibration sense below the left knee. The tendon reflexes in the lower limbs were brisk and the plantar responses were both extensor. A motor unit count revealed severe depletion of the hypothenar population (42 units) which could not have been due to a generalized motoneuronopathy (for example, peroneal muscular atrophy), or to a polyneuropathy, since the EDB motor unit count was normal (156 units). For this reason a cervical cord lesion involving the $\mathrm{C} 8$ and T1 segments seemed probable. The lesion was unlikely to have involved the cervical roots, since normal-sized sensory nerve potentials could be recorded from the digital branches of the median and ulnar nerves to the ring and little fingers (also supplied by (8). The conclusion drawn was that the lesion was situated ventrally within the cord and the tentative diagnosis of syringomyelia, based on the electrophysiological findings alone, was subsequently confirmed radiologically.

\section{DISCUSSION}

The conventional method for detecting denervation is to analyse the density of the interference pattern recorded with a concentric needle electrode from a muscle undergoing a maximal voluntary contraction. One problem with this method is that it depends on the full cooperation of the subject and therefore cannot be used in those with impaired consciousness or in malingering or hysterical patients. Other patients are sometimes unwilling to make a full contraction because of increasing pain at the site of the needle. The use of a concentric needle electrode presents another difficulty, for the inner electrode will be able to record from only a small number of units in its immediate vicinity and even then very few fibres of each unit will be sampled. Again, it is well known that not only the amplitude but also the complexity of a motor unit potential may be altered by small movements of the electrode. Finally, Stalberg (1972) has recently shown that individual motor unit potentials in diseased muscles may have durations as long as $40 \mathrm{msec}$ so that, given a discharge frequency of $25 / \mathrm{sec}$ or higher, a single motor unit can generate continuous electrical activity without any pauses between successive complexes.

In the quantal method described by McComas et al. (1971d), a different approach was used, in which the mean amplitude of a sample of motor unit potentials was compared with the maximum evoked response of the same muscle. Unlike interference pattern electromyography, the quantal method could be applied to virtually any patient. In addition, the relatively large surface electrodes which were employed in the latter technique undoubtedly gave better estimates of motor unit sizes. Above all, the quantal method provided a numerical value for the motor unit population in the muscle under investigation. In the original study by McComas et al. (1971d) the method was restricted to the extensor digitorum brevis (EDB) muscle; now, for reasons presented earlier (see the introductory section), it has been extended to the hypothenar muscles and median-innervated thenar ones. The accuracy of the estimates obtained by the quantal method is severely limited by several factors-the small number of motor units which can be sampled in any one subject, the large differences in the sizes of motor units, the occurrence of 'alternation' and the varying distances between the active units and recording electrodes. Of these factors the only one which interferes with the actual investigation is alternation; in about $5 \%$ of control subjects this was so marked in the thenar and 
hypothenar muscles that the technique could not be applied. Fortunately, the accuracy of the quantal method improves when partially denervated muscles are studied for the sample of units becomes a larger fraction of the total population. In addition, the chances of alternation are less and, in a chronic denervating process, the large increments are more easily distinguished from each other (see also McComas et al., 1971e). Examples of the hypothenar and thenar motor unit estimates in patients known to have denervating diseases have been included in the present study; in each case it was found that the estimates lay well outside the normal range.

It is instructive to compare the present results, determined by an electrophysiological method, with anatomical studies of muscle innervation. At the present time the axons supplying each of the thenar and hypothenar muscles do not appear to have been counted in man but Feinstein et al. (1955) have obtained values for two other intrinsic muscles of the hand. If half of the largest axons in the muscle nerves are alpha-motor (Cooper, 1966) then, from the data of Feinstein et al. (1955), the first dorsal interosseous muscle would contain about 100 motor units and the first lumbrical 80 . These values are reasonably close to those determined by dividing the hypothenar and thenar motor unit populations by the number of muscles contributing to the evoked responses. For the $2 \frac{1}{2}$ thenar muscles innervated by the median nerve-that is, abductor pollicis brevis, opponens pollicis, and part of the flexor pollicis brevis-the total population was 340 units, giving a mean of 136 units for one muscle. Similarly, for the four hypothenar muscles (palmaris brevis, abductor, opponens, and flexor digiti minimi) the total estimate was 380 units so that the mean content for one muscle would be 95 units. These values are obviously very approximate, for the muscles in each group differ considerably from each other in size, and might therefore be expected to show corresponding differences in motor unit populations. In contrast with the preceding comparison of our results with those of Feinstein et al. (1955), there seems to be no agreement with the findings of Christensen (1959). This author studied the muscles of stillborn infants and estimated that the opponens pollicis alone contained about 6,000 motor units. Although this value is far larger than those reported by Feinstein et al. (1955) for other intrinsic muscles of the hand, the reason for this large discrepancy is uncertain. It is of interest that the mean number of motor units obtained in the present study for the thenar muscles was rather larger than the value of $253 \pm 34$ recently published by Brown (1972) for the same group of muscles. This author used a quantal approach as well but differed in recording evoked activity with two small focal electrodes (Copeland-Davies 'clip-on' type). It is clear from Brown's published figures that the increments in the evoked response could readily be distinguished. Nevertheless, it is probable that these electrodes, because of their positions and small surfaces, would have recorded the largest potential increments from the abductor pollicis brevis (APB) muscle lying underneath them. In subjects in whom the APB motor axons had the lowest thresholds to electrical stimulation, the estimated number of units would have been small and weighted in favour of this muscle rather than the whole median-innervated thenar group. For this reason we feel that the use of a larger electrode may help to minimize distortions in increment size due to the different positions of the active units within the thenar eminence.

In the present study the results in older subjects are of special significance in relation to the nature of the mechanism responsible for muscle wasting and weakness in the elderly. Strong evidence favouring a neural aetiology was recently brought forward by Campbell and McComas (1970) and by Campbell et al. (1973), on the basis of diminished motor unit estimates in the extensor digitorum brevis (EDB) muscle. We have now been able to show that there is also a progressive loss of functioning motor units in small muscles of the hand and Brown (1972) has published similar findings for the thenar muscles. As with EDB it appears that there is little loss before 60 years of age but that by the ninth decade the number of units has fallen to less than half. In Fig. 2 of Brown's paper the numbers of units have been shown as a linear function of age throughout the age spectrum involved (13 to 89 years). This interpretation may have been incorrect, however, for little denervation appears to have taken place in his subjects under the age of 60 years. In the present study it was noted that the average sizes of the potentials generated by the surviving 
thenar and hypothenar motor units were significantly larger in the elderly subjects than in the younger ones. This observation suggested that, in the partially denervated muscles of elderly subjects, the motor units which were still functioning contained increased numbers of muscle fibres. This situation would have arisen if the motoneurones had retained some capacity for reinnervating denervated muscle fibres through axonal sprouting. A similar conclusion regarding EDB motoneurones in the elderly has been reported previously (Campbell and McComas, 1970; Campbell et al., 1973). In Brown's (1972) study the relationship between sizes and numbers of units for his elderly population was represented by a linear regression equation. A better fit for his experimental points would have been obtained with a hyperbolic curve and it is this type of relationship which would have been predicted if the sizes of the surviving units were proportional to the numbers of muscle fibres available for reinnervation (see also McComas et al., 1973). It is of interest that the present study suggests that the thenar motoneurones, for reasons that are not apparent, are unable to enlarge their muscle fibre population to quite the same extent as the hypothenar motoneurones. In younger subjects with partial denervation, however, very large motor units can be formed in the thenar muscles, as this investigation has shown.

We gratefully acknowledge financial support from the Canadian Medical Research Council; Dr. McComas is a member of the Council's Developmental Neurobiology Group and Dr. Sica held a Post-Doctoral Fellowship. We wish to thank those who volunteered as subjects for this study, and especially the residents of St. Joseph's Villa, Dundas, Ontario; we are also most grateful to Sister Mary Grace and Mr. K. Supple for their interest and organizational flair. We are also indebted to our clinical colleagues for their kindness in allowing us to study patients under their care and for their permission to publish some of the electrophysiological findings. Irene Csatari, Bernice Crompton, Joan English, Marianne Kozlo, Trevor Blogg, and Stephen Fielding provided skilled assistance. Judith Leon (funded by the Canadian Muscular Dystrophy Association) assisted in the preparation of the statistics and manuscript. Illustrations were made by Wendy Sawyer.

\section{REFERENCES}

Brown, W. F. (1972). A method for estimating the number of motor units in thenar muscles and the changes in motor unit count with ageing. Journal of Neurology, Neurosurgery, and Psychiatry, 35, 845-852.

Campbell, M. J., and McComas, A. J. (1970). The effects of ageing on muscle function. Fifth Symposium on Current Research in Muscular Dystrophy and Related Disorders, London (Abstracts). Muscular Dystrophy Group of Great Britain: London.

Campbell, M. J., McComas, A. J., and Petito, F. (1973). Physiological changes in ageing muscles. Journal of Neurology, Neurosurgery, and Psychiatry, 36, 174-182.

Christensen, E. (1959). Topography of terminal motor innervation in striated muscles from stillborn infants. American Journal of Physical Medicine, 38, 65-78.

Cooper, S. (1966). Muscle spindles and motor units. In Control and Innervation of Skeletal Muscle, pp. 9-17. Edited by B. L. Andrew, Livingstone: Edinburgh.

Feinstein, B., Lindegaard, B., Nyman, E., and Wohlfart, G. (1955). Morphologic studies of motor units in normal human muscles. Acta Anatomica (Basel), 23, 127-142.

Hayward, M. and Willison, R. G. (1973). The recognition of myogenic and neurogenic lesions by quantitative EMG. In New Developments in Electromyography and Clinical Neurophysiology, Vol. 2, pp. 448-453. Edited by J. Desmedt, Karger: Basel.

Jennekens, F. G. I., Tomlinson, B. E., and Walton, J. N. (1972). The extensor digitorum brevis: histological and histochemical aspects. Journal of Neurology, Neurosurgery, and Psychiatry, 35, 124-132.

McComas, A. J., Campbell, M. J., and Sica, R. E. P. (1971a). Electrophysiological study of dystrophia myotonica. Journal of Neurology, Neurosurgery, and Psychiatry, 34, 132-139.

McComas, A. J., Sica, R. E. P., and Brown, J. C. (1971b). Myasthenia gravis: evidence for a 'central' defect. Journal of the Neurological Sciences, 13, 107-113.

McComas, A. J., Sica, R. E. P., and Currie, S. (1971c). An electrophysiological study of Duchenne dystrophy. Journal of Neurology, Neurosurgery, and Psychiatry, 34, 461-468.

McComas, A. J., Fawcett, P. R. W., Campbell, M. J., and Sica, R. E. P. (1971d). Electrophysiological estimation of the number of motor units within a human muscle. Journal of Neurology, Neurosurgery, and Psychiatry, 34, 121-131.

McComas, A. J., Sica, R. E. P., Campbell, M. J., and Upton, A. R. M. (1971e). Functional compensation in partially denervated muscles. Journal of Neurology, Neurosurgery, and Psychiatry, 34, 453-460.

McComas, A. J., Sica, R. E. P., Upton, A. R. M., and Petito, F. (1973). Sick motoneurones and muscle diseases. Annals of the New York Academy of Sciences. (In press.)

Sica, R. E. P., and McComas, A. J. (1971). An electrophysiological investigation of limb-girdle and facioscapulohumeral dystrophy. Journal of Neurology, Neurosurgery, and Psychiatry, 34, 469-474.

Stalberg, E. Personal communication. 